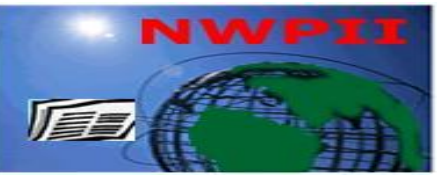

American Journal of Biomedical Sciences

ISSN: 1937-9080

nwpii.com/ajbms

\title{
Laser Surface Modification of Poly (E-caprolactone) Scaffold for Artificial Skin Applications
}

\author{
Gamal A. Abdel Hakeam ${ }^{1}$, Kamel Eid ${ }^{2}$, Mohammed A. Sharaf ${ }^{3}$, Yehia A. Badr ${ }^{1}$, \\ Marwa M. S. Abbass ${ }^{4 *}$, Nahed H. Solouma ${ }^{1}$ \\ ${ }^{l}$ National Institute of Laser Enhanced Sciences, Cairo University, Egypt \\ ${ }^{2,3}$ Department of Chemistry, Helwan University, Egypt \\ ${ }^{4}$ Faculty of Oral and Dental Medicine, Cairo University, Egypt \\ *Corresponding Author: \\ Marwa Magdy Abbass, Ph.D. \\ Department of Oral Biology \\ Faculty of Oral and Dental Medicine \\ Cairo University \\ Cairo, Egypt \\ Email: gamalamin@hotmail.com
}

Received:30 May 2012; | Revised:31 August 2012; | Accepted: 22 September 2012

\begin{abstract}
Hard implants undergo detachment from the host tissues due to inadequate biocompatibility, poor adhesion and subsequent cell integration. Thereby, surface engineering seems to offer solutions for improved functionality and biocompatibility of material implants inside the biological environment. Polycaprolactone (PCL) thin film has been fabricated via uniaxial compression technique. Pulsed excimer laser was used to modify the PCL surface roughness. The laser pulses induced the formation of nanoripples on the membrane surface. The effect of laser parameters (pulse rate, energy and number of pulses) on the development of the nanoripples was studied. The surface morphology, roughness and the scaffold biocompatibility and cell viability were characterized using scanning electron microscopes (SEM), atomic force microscope (AFM) and MTT assay micro-plate reader. This work showed that applying laser pulses at different rates significantly modified the surface criteria. The modified scaffold was more biocompatible, rough, with enhanced cell attachment, proliferation and provided adequate host for cells to differentiate rather than the unmodified scaffold. The results clearly revealed that the modified scaffold with nanoripples on its surface could be a candidate implant material for artificial skin applications.
\end{abstract}

Keywords: Surface engineering, PCL, Surface roughness, Laser. 


\section{Introduction}

Recently, Tissue engineering (TE) is gaining lots of attention as an emerging discipline that is advancing rapidly [1]. Medical applications can greatly benefit out of this development as tissue engineering represents a good alternative for biological substitutes in medical applications [2]. TE holds promises of eliminating re-operations by using biological substitutes [3], solves problems of implant rejection [4], transmission of diseases associated with xeno-grafts and allografts, and shortage in organ donation [5-6]. Furthermore TE provides long-term solutions in tissue repair [7], and potentially offers treatments for medical conditions that are currently untreatable[8-11]. Development of suitable biodegradable materials and scaffolds for seeding cells is the key of tissue engineering. Developing scaffolds is very challenging as they should be biocompatible[12], biodegradable [12] and provides good cell adhesion [13]. Tissue engineering is critical in need to ideal scaffold material and design [13-15]. Scaffolds can be prepared by either chemical or physical techniques. Chemical techniques include foaming[16], freeze drying[17] and phase separation[18]. Whereas, physical techniques are based on laser sintering[19], and rapid prototype machining [20]. There are lots of current limitation for the chemical methods due to the high impurity, lack of producing ideal scaffold for in-vivo applications[21]. On the other hand, physical treatments to the surface using laser ablation [23], plasma [24] or UV photo-curing [22] seems to offer solutions for improved functionality and biocompatibility of material implants inside the biological environment[23].

The laser-assisted modifications of polymer surfaces offer advantages over both chemical and other physical methods. They enable precise modification of certain surfaces that are difficult to be treated with conventional other methods. The resulting modified surfaces are free from contamination. They also could improve functionality and biocompatibility of materials which subsequently could be active implants inside the biological environment [24-28].
Synthetic and natural polymers are used for drug delivery $[29,30,31]$ and tissue engineering applications[32]. One of the synthetic polymers is PCL which posses many advantages. PCL is semi-crystalline linear resorpable aliphatic polyester[33]. It undergoes biodegradation[34] due to the susceptibility of its aliphatic ester linkage to hydrolysis. At present, PCL is regarded as a soft and hard tissue compatible bioresorpable material and it has been considered as a potential substrate for a wide spectrum of applications, such as drug delivery systems[35], bone tissue engineering [36], engineered skin[37], axonal regeneration, and scaffolds for supporting cell growth such as fibroblasts and osteoblasts [3840].

Herein, the surfaces of PCL scaffolds have been modified via laser treatments with varying the laser parameters such as energy, pulse rate and number of pulses. The effects of these parameters on the surface morphology, cell viability and differentiation was studied and characterized by SEM, AFM and MTT assay micro plate reader.

\section{Experimatal setup}

\subsection{Materials}

Poly ( $\varepsilon$-caprolactone) (average molecular weight ( $\mathrm{Mw})$ 60,000), Dulbecco's Modified Eagles Media (DMEM), glutamine, penicillin, Streptomycin, Ethanol HPLC and MTT dye [3(4,5-dimethyl thiazol-2-yl)-2,5-diphenyl tetrazolium bromide] were provided by Sigma Aldrich, Chemie GmbH (Munich, Germany). Phosphate buffer saline (PBS) and Polycarbonate filter paper were provided by El-gomhoria Co. Cairo, Egypt. Double deionized water from a Milli-Q ${ }^{\mathrm{TM}}$ system (Direct-Q 3, Model ZRQSOP0WW, Millipore Corporation, Billerica, MA) with a resistivity of $18 \mathrm{M} \Omega \mathrm{cm}$. All purchased Chemicals were used without additional purification.

\subsection{PCL membranes preparation}

PCL membranes have been fabricated by uniaxial compression technique; briefly PCL granules were heat pressed in a steel mould covered with a copper sheet (in order to equalize 
heat distribution) using a heat controlled piston at $60^{\circ} \mathrm{C}$ for 30 minutes. Then the steel mould was quenched in iced water to obtain a compressed and free of bubbles PCL bar with a dimension of $(3 \times 5 \times 20) \mathrm{mm}$. The PCL bar was heat pressed and quenched subsequently in iced water in a glass mould with the previous conditions to produce a PCL membrane of a thickness of $0.1 \mathrm{~mm}$.

\subsection{PCL scaffold modification with excimer laser}

Excimer laser pulses have been generated from a Lambda Physic Compex 205 system having krypton fluoride $(\mathrm{KrF})$ as the lasing gas, resulting in a laser wave length of $248 \mathrm{~nm}$, a band width of $300 \mathrm{pm}$ and a pulse duration of $24 \mathrm{~ns}$. The output pulse has a rectangular transverse cross section with a width of $0.6 \mathrm{~cm}$ and a height of $2.4 \mathrm{~cm}$. The beam intensity has a gaussian distribution in the vertical and horizontal directions. A beam guiding system has been used to reshape the pulse wave front into a square of $1.6 \times 1.6 \mathrm{~cm} 2$ and to homogenize the intensity of the beam in the transverse direction. The beam spot size on the target is $0.48 \times 0.48 \mathrm{~cm} 2$. The pulse energy density has been varied from 15 to $25 \mathrm{~mJ} / \mathrm{cm} 2$, the number of pulses has been changed from 500 to 2500 whereas the repetition rate has been changed from 2 to $5 \mathrm{~Hz}$. The treatments have been conducted under standard atmospheric conditions.

\section{Characterization of the scaffold}

\subsection{SEM imaging}

The microstructure and morphology of the scaffold have been examined using field emission scanning electron microscope (SEM, LEO SUPRA 55; Carl Zeiss AG, Oberkochen, Germany) at an accelerating voltage of $8-12 \mathrm{kV}$ (SEM) and with magnification in the range of 50$400 \mathrm{Kx}$. Briefly the samples were bleached, mounted on carbon tab and coated with gold using coated sputter (S150A Edward, England) and examined.

\subsection{Cell scaffold interaction}

Modified and unmodified PCL films were cut into 0.7 inch diameter circles. One film was cut to about 9 small substrates. Scaffolds of size $7 \mathrm{~mm} \times 7 \mathrm{~mm}$ were seeded in culture medium with human Fibroblast cells in 24 well plates for a period of 14 days. Briefly the scaffolds were submerged in $70 \%$ ethanol for 15 minute, sterilized with carbon dioxide at $50^{\circ} \mathrm{C}$ for one hour. They were then washed with PBS (7.4) and were soaked in $5 \mathrm{ml}$ of culture medium for $1 \mathrm{~h}$. The cells were grown in the plates at a density of $5 \times 106$ cells $/ \mathrm{ml}$ in Medium (DMEM) containing fetal bovine serum $(10 \% \mathrm{~V} / \mathrm{V})$ glutamine $(2 \mathrm{mM})$, penicillin $(100 \mathrm{U} / \mathrm{ml})$ and streptomycin $(1 \mu \mathrm{g} / \mathrm{ml})$ and incubated in $5 \% \mathrm{CO} 2$ at $37^{\circ} \mathrm{C}$. The culture media were changed every 48 hours for a period of 14 days. The experiment was repeated 3 times to estimate the standard deviation.

\subsection{In-vitro biological testing imaging}

The cell viability, cell proliferation and cell adhesion were evaluated via scanning electron microscope. The cell seeded scaffolds on days 3, 7, and14 were prepared for SEM observation by changing culture medium, washing 3 times in prewarmed PBS (7.4). After washing with PBS, the specimens were fixed in $2.5 \%$ glutaraldehyde at $40 \mathrm{C}$ for 4 hours and dehydrated in ascending grades of ethanol changes (50, 70, 90 and 97\%), each step lasting $30 \mathrm{~min}$. Then the samples were left to dry for two hours, mounted on aluminum stubs coated with carbon tape and examined by environmental scanning electron microscope under 6-10 kV (SEM).

\subsection{Roughness determination}

The surface roughness of the scaffolds has been examined and measured using atomic force microscope (Veeco Metrology, Plainview, NY, USA).

\subsection{In-vitro degradation test of the scaffold}

In-vitro degradation of the scaffolds was investigated by soaking in simulated body fluid (SBF) (PH 7.4) at 370C for 10 days. The ratio of solution volume to scaffold mass was $200 \mathrm{ml} / \mathrm{g}$. After soaking, the scaffolds was dried at $30^{\circ} \mathrm{C}$ for 1 day and the rate of weight loss of the scaffolds with different PCL contents as a function of soaking time in SBF were measured. The 
experiment was repeated 3 times to estimate the standard deviation.

\section{Results and Discussions}

\subsection{SEM imaging of the scaffold}

The morphology of the modified and unmodified PCL scaffold was shown in Figure 1 (A-F). In Figure 1(A-B) the surface of the scaffold was modified by changing the pulse rate (PR) $5 \mathrm{~Hz}(\mathrm{~A})$ and $3 \mathrm{~Hz}(\mathrm{~B})$. The varied $\mathrm{PR}$ lead to the formation of highly distributed, regular ripples on the scaffold surface with size ranged from 50-200nm in width and extended to 0.6$2 \mu \mathrm{m}$ in height. In Figure 1 (C-D) the surface of the scaffolds were modified by varying the number of pulses particularly 500(C) and 1500 (D). The ripples obtained in this case had size in the range from $20-150 \mathrm{~nm}$ in width and 200$800 \mathrm{~nm}$ in length. The ripples obtained by changing the energy density in the range of $20 \mathrm{~mJ} / \mathrm{cm} 2$ were shown in Figure $1(\mathrm{~F})$. They had size ranged from $200-500 \mathrm{~nm}$ in width and $1-2 \mu \mathrm{m}$ in length. In contrary, the surface had rough surface with rippled ripples with lack of deep ripples by using energy density in the range of $25 \mathrm{~mJ} / \mathrm{cm} 2$ Figure 1 (E). The SEM results revealed that the surface of PCL gained highly ordered, distributed ripples by changing the laser parameters particularly (energy density, pulse rate and number of pulses). In addition, the pulse rate had the greatest effect on the surface morphology. This data was in agreement with the data reported before for surface engineering via laser ablation and its ability to change surface morphology [41-42]. In the present study, the effect of excimer laser on the surface morphology have been studied to obtain the best rough surface to be used as a substrate for artificial skin.

Formation of these ripples in this study could be attributed to the fact that using a short pulse, medium laser intensity and lower number of pulses results in disintegration of PCL scaffold at a much faster rate and before the melt could land on the membrane surface that have already been disintegrated by the oncoming pulses [43]. In the modified scaffold by laser ablation, energy has to be delivered in excess of the binding energy of the atom so as to remove the atom from the solid or change chemical composition of these atoms. PCL melting point is very low $\left(65^{\circ} \mathrm{C}\right)$, thus, to modify the surface of the scaffold via changing the chemical composition we applied a short pulse, medium laser intensity, and this fluence is approximately inversely proportional to the pulse duration[44]. The fluence is able to ionize almost any target material that takes place early in the laser pulse time. Following ionization, the laser energy is absorbed by free electrons[45]. These energetic electrons break free from the bulk material and create a strong electric field due to charge separation from the parent ions. The magnitude of this electric field pulls the ions out of the solid target when the electron energy is larger than the binding energy of the ions, thus resulting in material removal[46]. In laser ablation of the scaffolds, the heat diffusion into the scaffold material is not negligible and the energy loss into the sample is minimized. There were many factors that could affect the result of the ripple perforation. First, the pulse energy (PE) of the laser could directly affect the size of the ripples[47-48]. A higher PE generally leads to scaffold melting and form larger craters. Next, the pulse number (PN) can affect the quality of the produced ripples, as low pulses generate greater ejections of melt on the scaffold, which subsequently leads to higher melting degree of the scaffold. It is clearly indicated that the PCL scaffold with ripples perforated at lower pulse numbers and had higher surface energy. At lower pulse numbers, the degree of surface roughness is higher because there were more surface asperities due to greater splattering effect. These asperities can influence the water droplet and particle interaction forces by modifying the Van der Waals interaction, which would then rupture the liquid membrane that holds the liquid together[49]. This rough surface is more suitable for cell growth and proliferation when implanted in the organs[45].

\subsection{Roughness analysis}

The average plane roughness of the scaffolds surface was monitored by AFM following excimer laser modification by changing the parameters (pulse rate, number of pulses and energy density). The surface roughness had 
values in the range of $150-320 \mathrm{~nm}$ by changing the number of pulses particularly $(500,1500$ and 2500) as shown in Figure 2. The surface was covered with distributed ripples varied in height and depth according to the change of the number of pulses which subsequently increased with increasing the number of pulses. The average surface roughness ranged from $50-280 \mathrm{~nm}$ by varying the energy density particularly $(15,20$ and $25 \mathrm{~mJ} / \mathrm{cm}^{2}$ ) as revealed in Figure 3. The ripples obtained in this case were disordered, with little number and shallow depth.
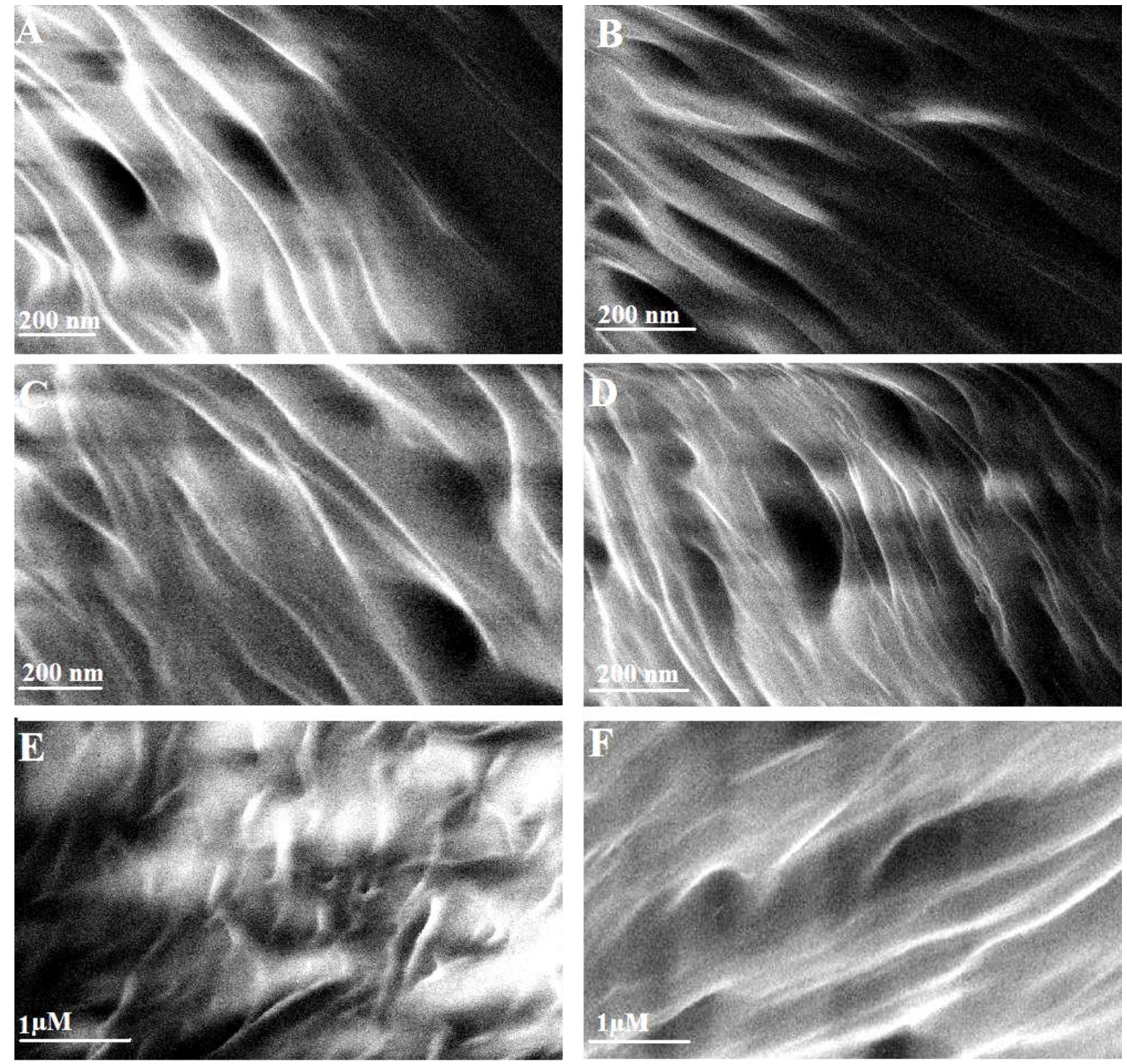

Figure 1 SEM images of modified scaffolds : (A-B) by using pulse rate 5,3 respectively with energy $60 \mathrm{~mJ} / \mathrm{cm}^{2}$, number of pulses 500, (C-D) by using pulse rate $3 \mathrm{~Hz}$ with energy $100 \mathrm{~mJ} / \mathrm{cm}^{2}$, number of pulses 500,1500 respectively and (E-F) by using pulse rate $3 \mathrm{~Hz}$, energy 25, 20 respectively $\mathrm{mJ} / \mathrm{cm}^{2}$ and number of pulses 1500 .

Figure 4 showed the surface roughness of PCL with varying the pulse rate particularly $(2,3$ and $5 \mathrm{~Hz}$ ). The surface had highly ordered, distributed and consecutive ripples varied in height and depth by changing the pulse rates. The AFM results clearly indicated that the surface roughness has been increased with changing the excimer laser parameters. It should be noted that 
the pulse rate had the major effect on the surface roughness. This data was in agreement with the SEM results which subsequently revealed that the laser treated PCL scaffolds provided good adhesive surface for cell attachment and differentiation. These results were in agreement with the data recorded before by Groenendijk, and Meijer 2006 who reported that laser parameters had the ability to change polymers' surface morphology[47].

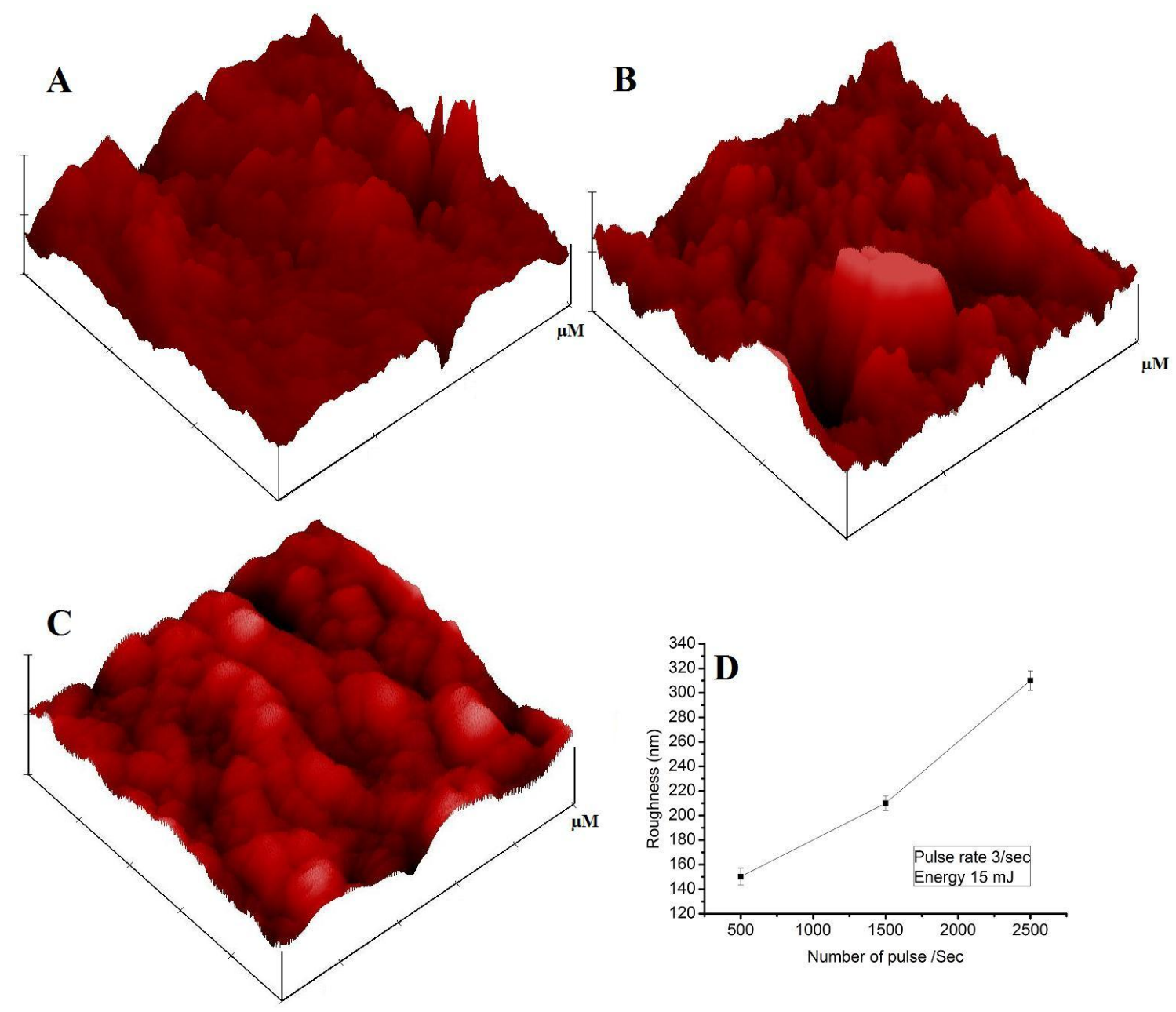

Figure 2 AFM of PCL scaffolds with varied of number of pulses $(500,1500$ and 2500) for A, B, and C respectively, energy density $15 \mathrm{~mJ} / \mathrm{cm}^{2}$ and pulse rate $3 \mathrm{~Hz}$.

\subsection{In-vitro degradation test}

The scaffold should be biodegradable and bioactive to support the cell differentiation and proliferation. The degradability of the modified and unmodified PCL scaffolds was monitored at 7, 14, 21 and 30 days in culture (Figure 5). Apparently, the degradation rate for both modified and unmodified PCL scaffolds increased gradually up till the day 30 . The results indicated that the degradation rate of the modified
PCL scaffold was greater than the unmodified scaffold. Laser excitation enhanced the crushability and thermal stability. The presence of nanoripples on the surface of the modified PCL scaffold increased the surface area of the scaffold, hence increasing their degradation. It should be noted that using alkaline medium or higher temperature accelerates the degradation rate of PCL scaffold. In the present work, the degradation rate was measured in SBF to 
simulate the in-vivo degradation. The results also clearly indicated that, the modified PCL scaffold, have good degradability in in-vitro bioactivity, more than the unmodified PCL scaffolds and it will have the ability to make a direct bond to living cells when implanted in the body. These results were in agreement with the results recorded before for degradation rates of PCL [5051].

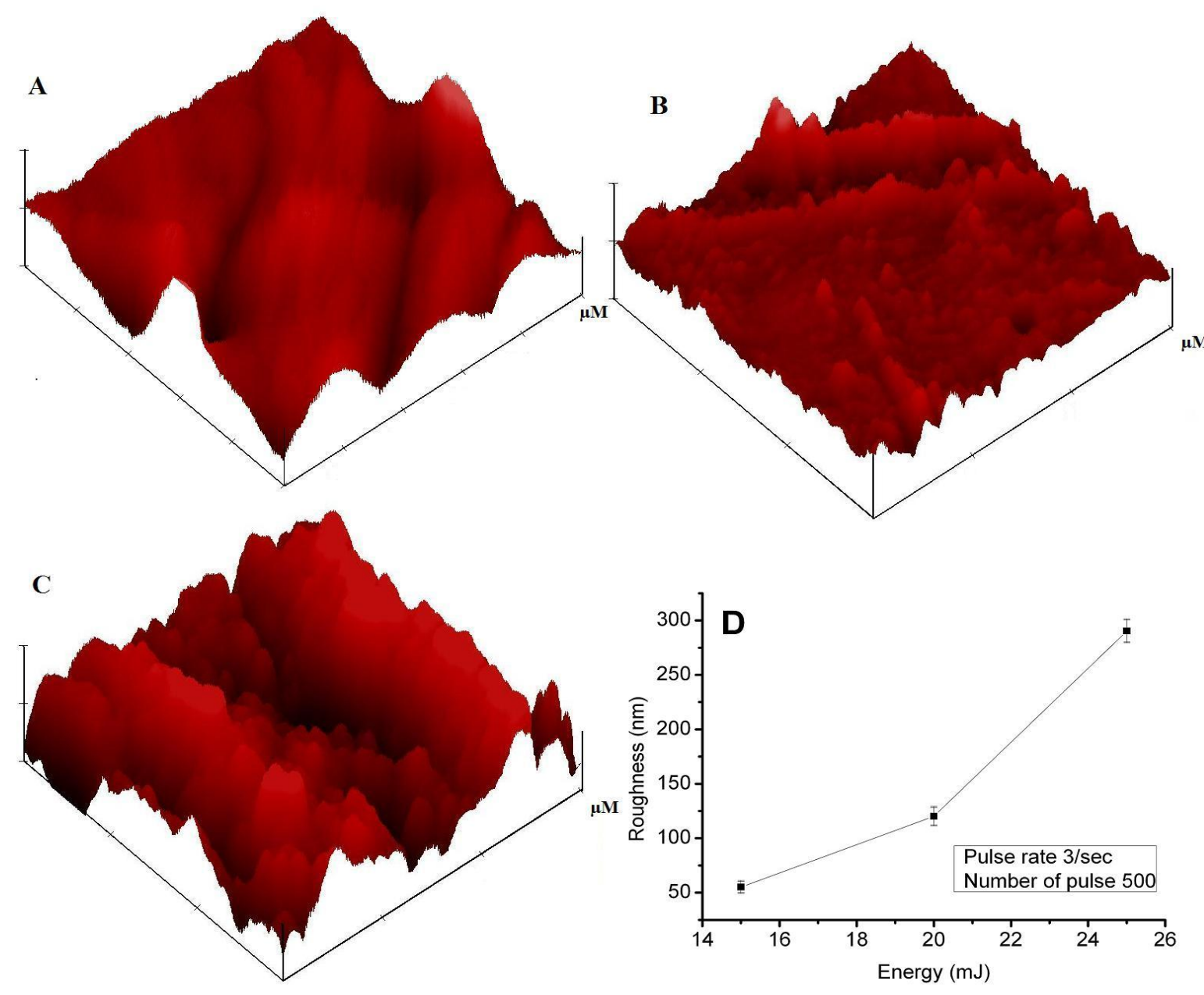

Figure 3 AFM of PCL scaffolds with varied energy density $\left(15,20\right.$ and $\left.25 \mathrm{~mJ} / \mathrm{cm}^{2}\right)$, number of pulses 500 and pulse rate $3 \mathrm{~Hz}$

\subsection{Cytotoxicity evaluation}

The quantitative cell viability on the modified and unmodified PCL scaffolds was evaluated using MTT assay with human fibroblast cells. In this assay, only cells that were viable after $24 \mathrm{~h}$ exposure to the PCL scaffolds were capable of metabolizing the dye efficiently and produce a purple colored precipitate which was analyzed and counted using a micro plate reader as shown in Figure 6. The fibroblasts showed excellent cell viability after 3 weeks of exposure to the modified PCL scaffolds relative to control cells. The number of cells were found to be approximately $3.1 \times 10^{6}, 5.6 \times 10^{6}$ and $7 \times$ $10^{6}$ alive cells per well after $1,2,3$ and 4 weeks of culturing. In contrary, the cells showed poor viability after 3 weeks of culturing on the unmodified PCL scaffold. As the number of cells were $1.9 \times 10^{6}, 1.5 \times 10^{6}$ and $1.0 \times 10^{6}$ after 1,2 and 3 weeks of culturing relative to controls. The MTT assay for the cell viability demonstrated that the modified PCL scaffold showed excellent cell viability rather than the unmodified scaffold. The rough surface for the modified PCL scaffold enhanced the cell viability as the cells were able to grow, differentiate and proliferate on the rough 
surface for the modified PCL scaffold. In contrary, the cells on the unmodified PCL scaffold undergo detachment due to inadequate

and poor adhesion. This data was in agreement with SEM and AFM result.

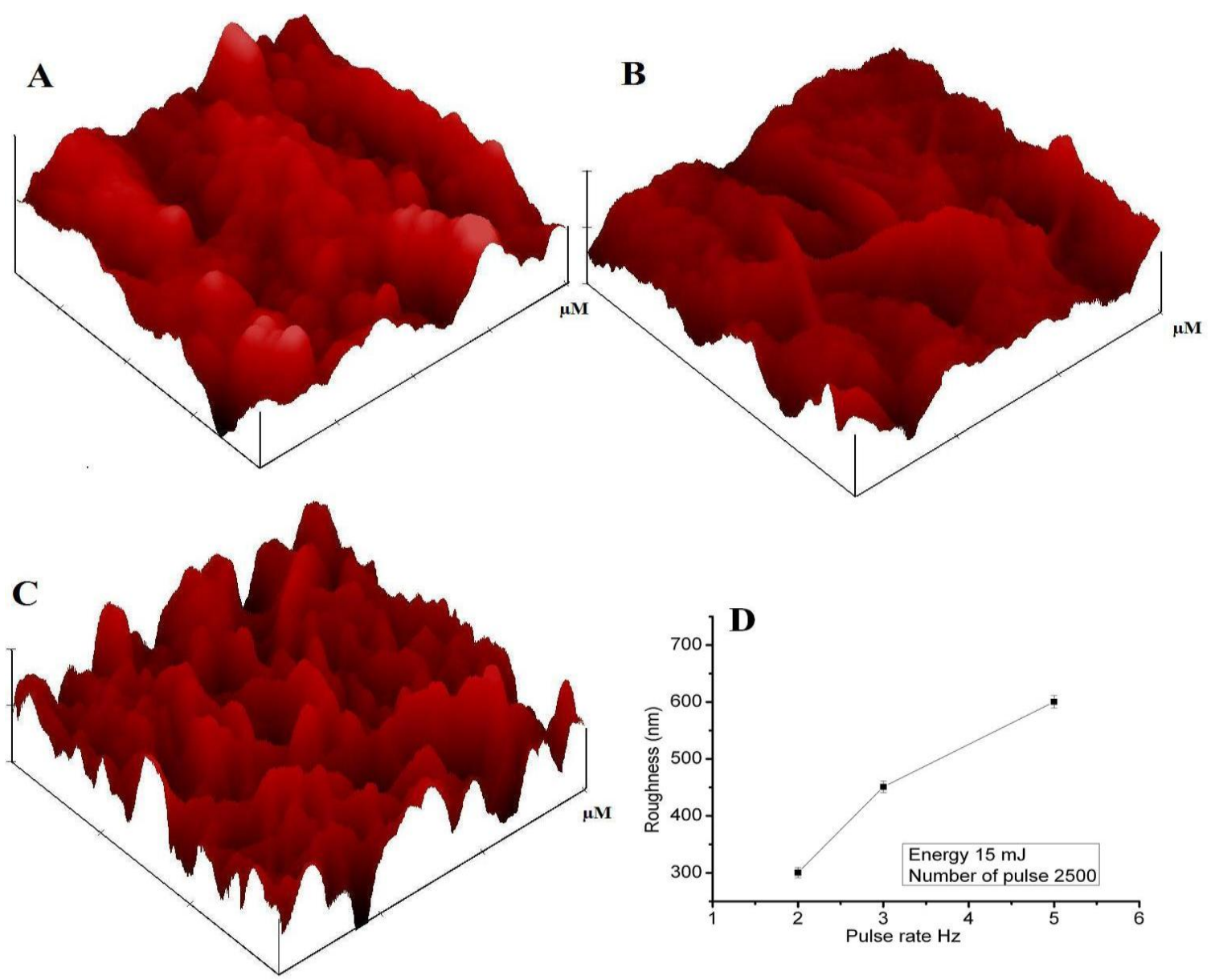

Figure 4 AFM of PCL scaffolds with varied pulse rate (2, 3 and 5Hz), number of pulses 2500 and energy density 15 $\mathrm{mJ} / \mathrm{cm}^{2}$.

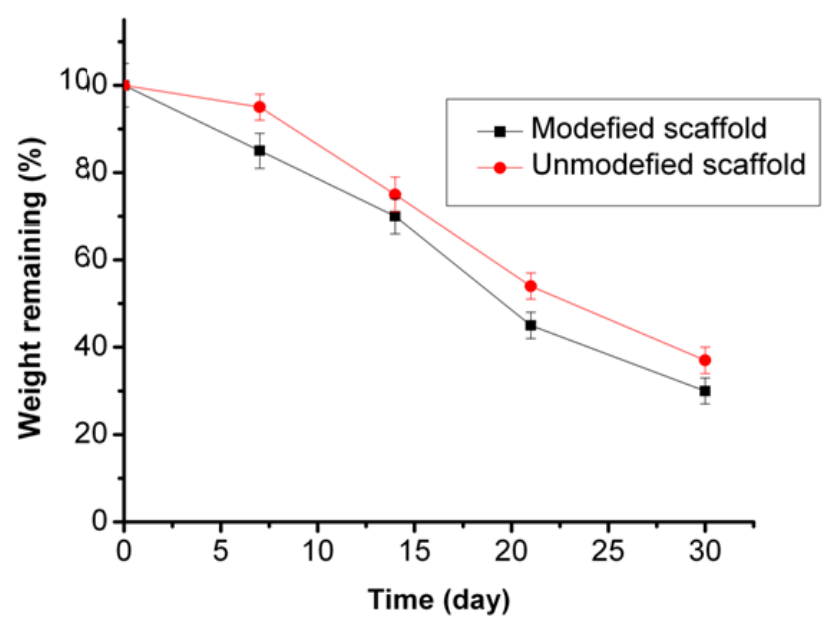

Figure 5 The degradation rate of unmodified and modified PCL scaffold that were prepared by using number of pulses 2500 and energy density $15 \mathrm{~mJ} / \mathrm{cm}^{2}$

\subsection{In-vitro biological cells imaging}

The morphology of cultured fibroblasts on the modified and unmodified PCL scaffolds was observed by SEM as shown in Figure 7. The cells growth and differentiation were monitored after 7, 14 days of culturing for both modified and unmodified PCL scaffolds. The fibroblasts cultured on the modified PCL scaffold have grown faster and proliferated to form elliptical shape after 7 days of culturing Figure 7 (A). After 14 days of culturing the fibroblasts were completely differentiated on the scaffold surface, formed a sheet of cells and matrix like structure Figure 7 (B). In contrary, the fibroblasts cultured on the unmodified PCL scaffolds showed a rod shape with smooth surface after 7 days of culturing Figure 7(C). 


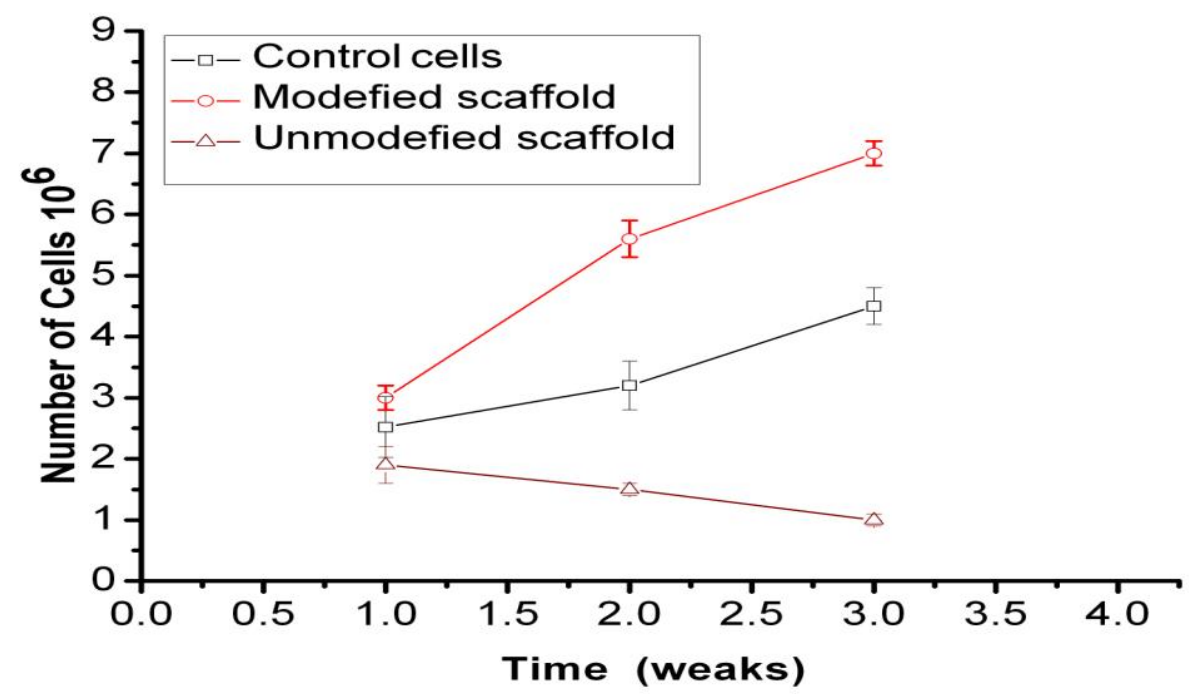

Figure 6 The MTT assay for the cell viability for unmodified and modified PCL scaffolds that were prepared by using number of pulses 2500 and energy density $15 \mathrm{~mJ} / \mathrm{cm} 2$ relative to control.
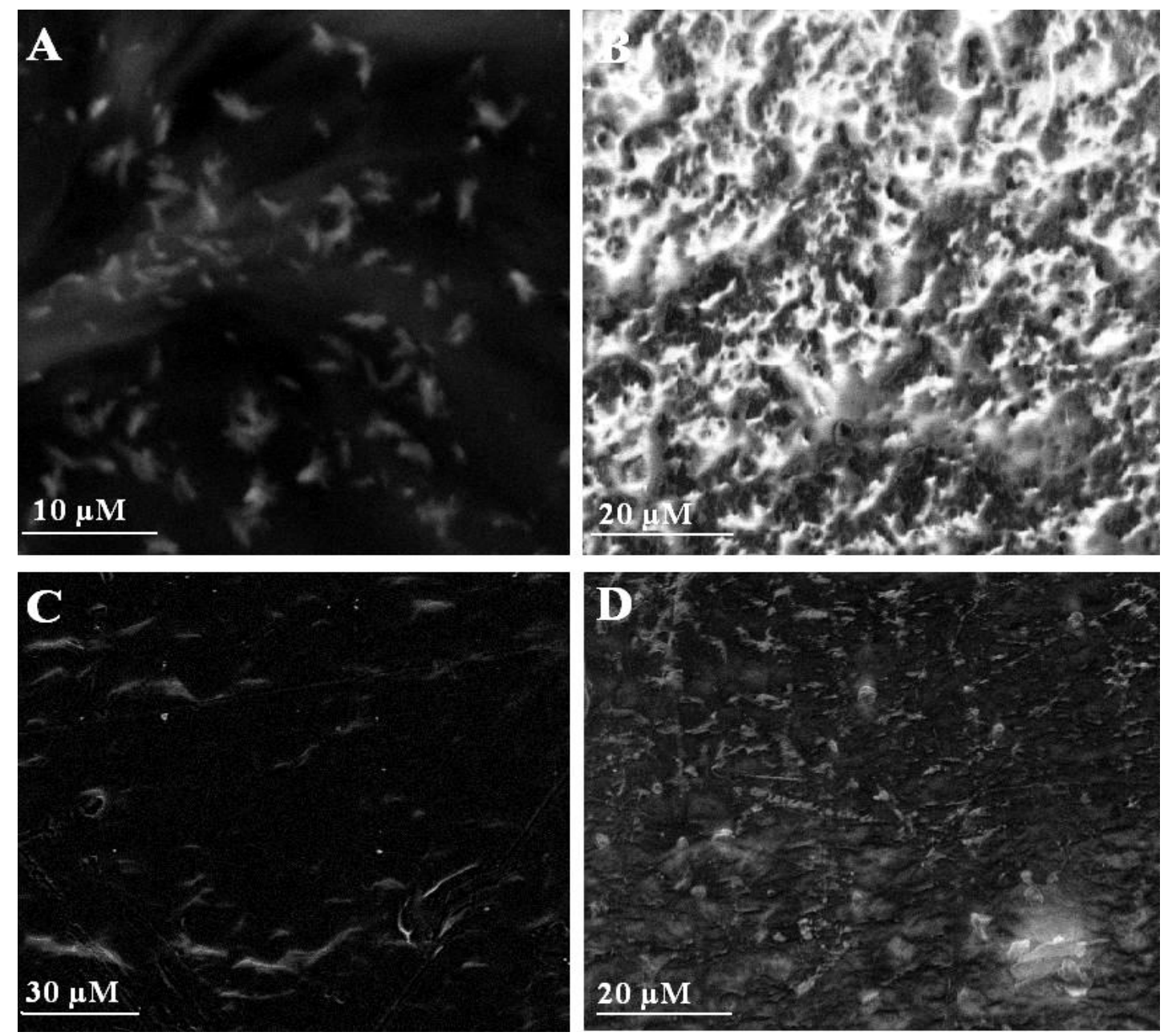

Figure 7 The cell morphology of human fibroblast cells on the modified PCL scaffold that were prepared by using number of pulses 2500 and energy density $15 \mathrm{~mJ} / \mathrm{cm}^{2}$ (A) after 7 days of culturing and (B) after 14 days of culturing. The cells morphology on the unmodified PCL scaffold after 7 days of culturing (C) and after 14 days of culturing (D). 
Then the cells elongated to longer rods and become spinal in shape with lack of any matrix or sheet of cells Figure 7 (D). This was due to poor attachment of cells on the scaffold surface.

From the results gathered from the cell culture, it was clearly indicated that the laser modified scaffolds were safe material and good carriers for cells as they promoted cell attachment, allowed cell proliferation and differentiation. Therefore, modified scaffolds provide candidate tools for artificial skin and tissue regeneration. These results were in agreement with the data reported before that modified PCL scaffold provided adequate substrate for cell adhesion and differentiation [52-53].

\section{Conclusions}

In this study, the effect of laser illumination parameters (pulse rate, number of pulses and energy density) on PCL scaffolds surface morphology, roughness, cell viability and cell adhesion were studied. These modified scaffolds could be used for tissue engineering application especially for artificial skin implants. The AFM results demonstrated that the pulse rate had the paramount effect on surface roughness and morphology. The most beneficial surface roughness was obtained with changing the pulse rate and had values ranged from 300 to $600 \mathrm{~nm}$. The modified PCL scaffold had good degradability in in-vitro bioactivity, more than the unmodified PCL scaffolds reached to $30 \%$ of the original weight. From In-vitro biological test imaging, the SEM results depicted good adhesion of cells on the modified scaffolds in comparison with the unmodified scaffold. The cells formed complete sheet like structure after 14 days of culturing with lack of any matrix or sheet like structure on the unmodified PCL scaffold. These results clearly indicated that the modified PCL scaffold provided adequate, sufficient surface for cells adhesion, proliferation and could have the ability to make direct bond to living cells when implanted in the body.

\section{References}

1. Andrianantoandro, E., et al., Synthetic biology: new engineering rules for an emerging discipline. Molecular systems biology, 2006. 2(1). doi:10.1038/msb4100073

2. Helder, M.N., et al., Stem cells from adipose tissue allow challenging new concepts for regenerative medicine. Tissue Engineering, $2007 . \quad 13(8)$ : p. 1799-1808. doi:10.1089/ten.2006.0165

3. Ingber, D.E., et al., Tissue engineering and developmental biology: going biomimetic. Tissue Engineering, 2006. 12(12): p. 32653283. doi:10.1089/ten.2006.12.3265.

4. Lanza, R.P. and J. Vacanti, Principles of tissue engineering. 2007: Academic Press.

5. Cebotari, S., et al., Clinical application of tissue engineered human heart valves using autologous progenitor cells. Circulation, 2006. 114(1 suppl): p. I-132-I-137. doi: 10.1161/CIRCULATIONAHA.105.001065

6. Macri, L. and R. Clark, Tissue engineering for cutaneous wounds: selecting the proper time and space for growth factors, cells and the extracellular matrix. Skin Pharmacol Physiol, 2009. 22(2): p. 83-93. doi: $\underline{10.1159 / 000178867}$

7. Kao, S.T. and D.D. Scott, A review of bone substitutes. Oral and maxillofacial surgery clinics of North America, 2007. 19(4): p. 513521. doi: 10.1016/j.coms.2007.06.002

8. Tang, Y., et al., Combination of bone tissue engineering and BMP-2 gene transfection promotes bone healing in osteoporotic rats. Cell biology international, 2008. 32(9): p. 1150-1157. doi: 10.1016/j.cellbi.2008.06.005

9. Salvadè, A., et al., GMP-grade preparation of biomimetic scaffolds with osteo-differentiated autologous mesenchymal stromal cells for the treatment of alveolar bone resorption in periodontal disease. Cytotherapy, 2007. 9(5): p. 427-438. doi:10.1080/14653240701341995

10. Mpamhanga, C.P., et al., One scaffold, three binding modes: novel and selective pteridine reductase 1 inhibitors derived from fragment hits discovered by virtual screening. Journal of medicinal chemistry, 2009. 52(14): p. 4454-4465. doi: 10.1021/jm900414x 
11. Gu, H., et al., Control of in vitro neural differentiation of mesenchymal stem cells in $3 D$ macroporous, cellulosic hydrogels. Regenerative medicine, 2010. 5(2): p. 245253. doi:10.2217/rme.09.89

12. Aly, A.F., et al., Scaffold Developmentand Characterization Using CAD System. Am. J. Biomed. Sci, 2011. 3(4): p. 268-277. doi: $\underline{10.5099 / a j 110400268}$

13. Tomlinson, C.A., et al., The parallel curriculum: A design to develop learner potential and challenge advanced learners. 2008: Corwin Pr.

14. Blackwood, K.A., et al., Development of biodegradable electrospun scaffolds for dermal replacement. Biomaterials, 2008. 29(21): p. 3091-3104.

15. Park, D.C. and P. Reuter-Lorenz, The adaptive brain: aging and neurocognitive scaffolding. Annual review of psychology, 2009. 60: p. 173-196. doi: 10.1146/annurev.psych.59.103006.093656

16. $\mathrm{Fu}, \mathrm{Q}$, et al., Mechanical and in vitro performance of 13-93 bioactive glass scaffolds prepared by a polymer foam replication technique. Acta Biomaterialia, 2008. 4(6): p. 1854-1864. doi: 10.1016/j.actbio.2008.04.019

17. Haugh, M.G., C.M. Murphy, and F.J. O'Brien, Novel Freeze-Drying Methods to Produce a Range of CollagenGlycosaminoglycan Scaffolds with Tailored Mean Pore Sizes. Tissue Engineering Part C: Methods, 2009. 16(5): p. 887-894. doi: 10.1089/ten.tec.2009.0422.

18. Liu, X. and P.X. Ma, Phase separation, pore structure, and properties of nanofibrous gelatin scaffolds. Biomaterials, 2009. 30(25): p. 4094-4103.

19. Yeong, W., et al., Porous polycaprolactone scaffold for cardiac tissue engineering fabricated by selective laser sintering. Acta Biomaterialia, 2010. 6(6): p. 2028-2034. doi: 10.1016/j.actbio.2009.12.033.

20. Uklejewski, R., et al., Selective laser melted prototype of original minimally invasive resurfacing hip endoprosthesis. Rapid Prototyping Journal, 2011. 17(1): p. 76-85. doi; $10.1108 / 13552541111098653$
21. O'Brien, F.J., Biomaterials \& scaffolds for tissue engineering. Materials Today, 2011. 14(3): p. 88-95. doi: 10.1016/S13697021(11)70058-X,

22. Brandi, F., et al., Rigid biodegradable photopolymer structures of high resolution using deep-UV laser photocuring. Journal of Micromechanics and Microengineering, 2011. 21: p. 054007. doi: 10.1088/0960$1317 / 21 / 5 / 054007$

23. Öteyaka, M., et al., Effect of Surface Modification by Ammonia Plasma on Vascular Graft: PET Film and PET Scaffold. Acta Physica Polonica-Series A General Physics. 2012;121:125.

24. Kanczler, J.M., et al., Biocompatibility and osteogenic potential of human fetal femurderived cells on surface selective laser sintered scaffolds. Acta Biomaterialia, 2009. 5(6): $\quad$ p. $2063-2071$. doi: 10.1016/j.actbio.2009.03.010

25. McCullen, S.D., et al., In situ collagen polymerization of layered cell-seeded electrospun scaffolds for bone tissue engineering applications. Tissue Engineering Part C: Methods, 2010. 16(5): p. 1095-1105. doi: 10.1089/ten.tec.2009.0753.

26. Elloumi - Hannachi, I., M. Yamato, and T. Okano, Cell sheet engineering: a unique nanotechnology for scaffold-free tissue reconstruction with clinical applications in regenerative medicine. Journal of internal medicine, 2010. 267(1): p. 54-70. doi: 10.1111/j.1365-2796.2009.02185.x

27. Nagase, K., J. Kobayashi, and T. Okano, Temperature-responsive intelligent interfaces for biomolecular separation and cell sheet engineering. Journal of The Royal Society Interface, 2009. 6(Suppl 3): p.S293-S309. doi: 10.1098/rsif.2008.0499.focus

28. Hasirci, V. and H. Kenar, Novel surface patterning approaches for tissue engineering and their effect on cell behavior. Nanomedicine, 2006. 1(1): p. 73-90. DOI 10.2217/17435889.1.1.73

29. Cho, K., et al., Therapeutic nanoparticles for drug delivery in cancer. Clinical Cancer Research, 2008. 14(5): p. 1310-1316. doi: 10.1158/1078-0432.CCR-07-1441 
30. Gils, P.S., D. Ray, and P.K. Sahoo, Controlled Release of Doxofylline from Biopolymer Based Hydrogels. Am. J. Biomed. Sci, 2010. 2(4): p. 373-383. doi: 10.5099/aj100400373

31. Shaw, J., et al., Synthesis and Biological Evaluation of Novel N-phenyl-5carboxamidyl Isoxazoles as Potential Chemotherapeutic Agents for Colon Cancer. Am. J. Biomed. Sci, 2012. 4(1): p. 14-25. doi: 10.5099/aj120100014

32. Lutolf, M. and J. Hubbell, Synthetic biomaterials as instructive extracellular microenvironments for morphogenesis in tissue engineering. Nature biotechnology, 2005. 23(1): p. 47-55. doi: 10.1038/nbt1055

33. Williams, J.M., et al., Bone tissue engineering using polycaprolactone scaffolds fabricated via selective laser sintering. Biomaterials, 2005. 26(23): p. 4817-4827. doi: 10.1016/j.biomaterials

34. Tay, F.R., et al., Susceptibility of a polycaprolactone-based root canal filling material to degradation. I. Alkaline hydrolysis. Journal of endodontics, 2005. 31(8): p. 593-598.

35. Lam, C.X.F., et al., Evaluation of polycaprolactone scaffold degradation for 6 months in vitro and in vivo. Journal of Biomedical Materials Research Part A, 2009. 90(3): $\quad$ p. $906-919$. DOI: $10.1002 / \mathrm{jbm} . \mathrm{a} \cdot 32052$

36. Kyriakidou, K., et al., Dynamic co-seeding of osteoblast and endothelial cells on $3 D$ polycaprolactone scaffolds for enhanced bone tissue engineering. Journal of Bioactive and Compatible Polymers, 2008. 23(3): p. 227. doi: $10.1177 / 0883911508091905$

37. Powell, H.M. and S.T. Boyce, Engineered human skin fabricated using electrospun collagen-PCL blends: morphogenesis and mechanical properties. Tissue Engineering Part A, 2009. 15(8): p. 2177-2187. doi:10.1089/ten.tea.2008.0473.

38. Tan, P. and S. Teoh, Effect of stiffness of polycaprolactone (PCL) membrane on cell proliferation. Materials Science and Engineering: C, 2007. 27(2): p. 304-308. doi: 10.1016/j.msec.2006.03.010
39. Tay, F.R., et al., Susceptibility of a polycaprolactone-based root canal filling material to degradation. II. Gravimetric evaluation of enzymatic hydrolysis. Journal of endodontics, 2005. 31(10): p. 737-741. doi: 10.1097/01.don.0000155225.40794.79

40. Yildirim, E.D., et al., Accelerated differentiation of osteoblast cells on polycaprolactone scaffolds driven by a combined effect of protein coating and plasma modification. Biofabrication, 2010. 2: p. 014109 . doi:10.1088/17585082/2/1/014109

41. Tiaw, K., et al., Laser surface modification of poly (-caprolactone)(PCL) membrane for tissue engineering applications. Biomaterials, 2005. 26(7): p. 763-769. doi:10.1016/j.biomaterials.2004.03.010

42. Talton, J.D., Method of pulsed laser assisted surface modification. 2003, US Patent App. 20,060/051,522.

43. Wong, W., et al., Surface structuring of poly (ethylene terephthalate) by $U V$ excimer laser. Journal of materials processing technology, 2003. 132(1): p. 114-118. doi: 10.1016/S0924-0136(02)00412-0

44. Weck, A., et al., Ripple formation during deep hole drilling in copper with ultrashort laser pulses. Applied Physics A: Materials Science \& Processing, 2007. 89(4): p. 10011003. DOI: $10.1007 / \mathrm{s} 00339-007-4203-6$

45. Brown, M.S. and C.B. Arnold, Fundamentals of Laser-Material Interaction and Application to Multiscale Surface Modification. Laser Precision Microfabrication, 2010: p. 91-120. DOI: 10.1007/978-3-642-10523-4_4

46. Groenendijk, M. and J. Meijer, Microstructuring using femtosecond pulsed laser ablation. Journal of Laser Applications, 2006. 18: p. 227.

47. Groenendijk, M. and J. Meijer, Surface microstructures obtained by femtosecond laser pulses. CIRP Annals-Manufacturing Technology, 2006. 55(1): p. 183-186. doi: 10.1016/S0007-8506

48. Richter, A., S.A. Mahmoud, and R. Ries, Changes of biofilm structures on polymer substrates caused by laser beams. Vacuum, 
2002. 66(2): p. 179-188. doi: $10.1016 / \mathrm{S} 0042-207$

49. Drummond, C.J. and D.Y.C. Chan, van der Waals interaction, surface free energies, and contact angles: dispersive polymers and liquids. Langmuir, 1997. 13(14): p. 38903895. DOI: 10.1021/la962131c

50. Lam, C.X.F., S.H. Teoh, and D.W. Hutmacher, Comparison of the degradation of polycaprolactone and polycaprolactone( $\beta$-tricalcium phosphate) scaffolds in alkaline medium. Polymer international, 2007. $\quad \mathbf{5 6}(6)$ : $\quad$ p. $\quad$ 718-728. DOI: $10.1002 /$ pi.2195

51. Sanchez, J.G., A. Tsuchii, and Y. Tokiwa, Degradation of polycaprolactone at $50^{\circ} \mathrm{C}$ by a thermotolerant Aspergillus sp.
Biotechnology Letters, 2000. 22(10): p. 849-853. DOI: 10.1023/A:1005603112688

52. Lim, Y.C., et al., Micropatterning and characterization of electrospun poly $(\varepsilon$-caprolactone)/gelatin nanofiber tissue scaffolds by femtosecond laser ablation for tissue engineering applications. Biotechnology and bioengineering, 2011. 108(1): p. 116-126. doi: 10.1002/bit.22914

53. He, L., et al., Wettability modification of electrospun poly (-caprolactone) fibers by femtosecond laser irradiation in different gas atmospheres. Applied Surface Science, 2010. doi: 10.1016/j.apsusc.2010.11.072 